\title{
Glioblastoma multiforme y estudio de la resistencia a la quimioterapia mediada por transportadores $\mathrm{ABC}$
}

\author{
CLAUDIA QUEZADA $^{1, \mathrm{a}}$, LILIA PEIGÑAN ${ }^{1, \mathrm{a}}$, RODRIGO SEGURA ${ }^{2}$, \\ FRANCISCO RIQUELME ${ }^{2}$, RÓMULO MELO ${ }^{2}$ DAVID ROJAS Z. ${ }^{2}$, \\ FREDDY AYACH ${ }^{2}$, RODY SAN MARTÍN ${ }^{1, a}$, JUAN GUILLERMO CÁRCAMO ${ }^{1, a}$
}

\section{Study of resistance to chemotherapy mediated by $\mathrm{ABC}$ transporters in biopsies of glioblastoma multiforme}

Background: Mortality rate is dramatically high in high-grade brain tumors. The presence of multiple drug resistance transporters in glioblastoma multiforme, has contributed largely to the poor efficacy of targeted therapy against cancer in the central nervous system. Aim: To analyze the percentage of survival and mortality of patients with glioblastoma multiforme in a cohort of patients in Chile and to correlate the chemo-resistance of these cells with the expression level of multiple drug resistance transporters. Materials and Methods: Eighteen biopsies of glioblastoma multiforme were obtained from patients at the Institute of Neurosurgery Dr. Asenjo (INCA). The tumor cells were obtained from primary cultures and the expression and activity of multiple drug resistance transporters was assessed by RT-PCR and immunohistochemistry. Population-based study was performed using the databases of the Department of Neurosurgery of INCA. Results: The number of patients with glioblastoma multiforme increased between 2007 and 2009, from 3.5\% to 7.9\% of total brain tumors. Mortality of these tumors is $90 \%$ at three years. A high expression and activity of the multiple drugs resistance associated protein 1 (Mrp1) transporter was observed in primary cultures of biopsies. Conclusions: We propose that Mrp1 activity is responsible for the chemo-resistance of the glioblastoma multiforme and inhibition of this transporter could represent a plausible strategy for the treatment.

(Rev Med Chile 2011; 139: 415-424).

Key words: Drug resistance, neoplasm; Glioblastoma; Multidrug resistanceassociated proteins.

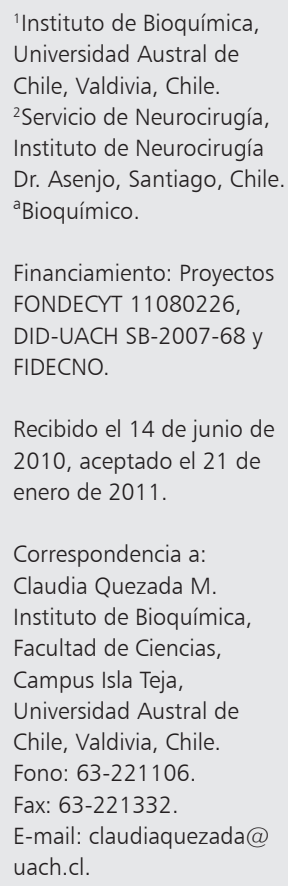

L as neoplasias son una de las enfermedades más frecuentes en el mundo y corresponden a la segunda causa de muerte en Chile, después de las enfermedades del sistema circulatorio según el Instituto Nacional de Estadisticas de Chile (Principales causas de muerte en Chile por regiones 1997-2003). La tasa de incidencia de los tumores cerebrales malignos y benignos del sistema nervioso central (SNC) en Estados Unidos de Norteamérica (EEUU) es de alrededor de 11,4 casos por 100.000 personas/año $(4,2$ por 100.000 persona/año para benigno y 6,8 por 100.000 persona/año para malignos). La mortalidad por tumores cerebrales representa el 2,4\% de las muertes por cáncer en EEUU; en niños es la segunda causa específica de muerte, después de las neoplasias hematológicas ${ }^{1}$. En Chile, la tasa de mortalidad por cáncer es de 119,4 por 100.000 representando el 23\% de todas las muertes; en tanto, la tasa de mortalidad por cáncer cerebral varía de 1 a 2 por 
100.000 con proyección al ascenso ${ }^{2}$. La incidencia de los tumores primarios de cerebro ha tenido un notorio incremento en las últimas décadas, esta tendencia seguirá aumentando, pronosticándose que cada año aproximadamente a 14.000 personas se les diagnosticará cáncer cerebral a nivel mundial ${ }^{3,4}$, siendo el pronóstico de vida de estos pacientes inferior a 24 meses $^{5,6}$.

La resistencia de las células cancerosas a fármacos antitumorales es una de las causas de fracaso en la quimioterapia del cáncer. Uno de los principales mecanismos involucrados en este fenotipo denominado resistencia múltiple a drogas (MDR), es la sobreexpresión de una o más proteínas de la familia de transportadores ABC ( $\underline{A} T P$ - $\underline{b}$ inding c cassette) en las células tumorales ${ }^{7}$, mientras que las células cerebrales expresan normalmente bajos niveles de algunos de estos transportadores ${ }^{8}$. Estas proteínas funcionan como bombas dependientes de ATP para la remoción de diversos metabolitos y xenobióticos desde la célula, entre los que destacan fármacos antitumorales. Existe un limitado número de estudios en tumores cerebrales de alto grado que identifiquen los subtipos de transportadores MDR, el nivel de expresión de ellos y además carecen de un análisis funcional y farmacológico detallado. Este desconocimiento ha contribuido en gran parte a la pobre eficacia de las terapias dirigidas al cáncer en el SNC. Cabe destacar que actualmente el manejo de los pacientes con tumores cerebrales es en primera instancia la extirpación, seguido de la quimioterapia, radioterapia, o ambas. Sin embargo, una importante limitación es que existe una restringida capacidad para extirparlos mediante cirugía, lo cual conlleva inevitablemente a la reincidencia y metástasis, disminuyendo notablemente la sobrevida de los pacientes. Por lo tanto, la mejor alternativa para el manejo de este tipo de cáncer es optimizar la quimioterapia. Esto implica mejorar la biodisponibilidad de los fármacos antitumorales en las células que conforman el glioma, así como también aumentar su acceso al cerebro, en donde la barrera hematoencefálica (BHE) podría restringir el paso de los agentes antitumorales ${ }^{8-10}$.

Aunque hay una gran cantidad de información disponible sobre las proteínas MDR, los tres principales transportadores de drogas $A B C$ que se cree que juegan un papel importante en la resistencia a los fármacos en las células cancerosas son, la Pglicoproteína (P-gp, Mdr1), la proteína 1 de múltiple resistencia a droga (Mrp1) y ABCg2 (BCRP) ${ }^{11}$.
Existen estudios, usando diferentes especímenes de gliomas y sus cultivos primarios, que demuestran un predominante contenido de miembros de la subfamilia MRP, particularmente Mrp1 pero bajos niveles de P-gp y otros transportadores $\mathrm{ABC}$ en gliomas de alto grado ${ }^{12-14}$.

Nuestro objetivo fue determinar el número y el porcentaje de mortalidad de pacientes con glioblastoma multiforme (Gbm) en el Instituto de Neurocirugía Asenjo de Santiago-Chile en los últimos años e identificar la sobreexpresión de Mrp1, P-gp o ABCg2 en cultivos primarios y tejidos tumorales de Gbm, con el objetivo de caracterizar cuál es el mecanismo de resistencia a la quimioterapia en nuestra población.

\section{Material y Métodos}

Las muestras fueron tratadas de acuerdo a las normas de bioética de la Universidad Austral de Chile, Valdivia y del Servicio de Salud Metropolitano Oriente, Santiago. El Servicio de Neurocirugía resecó y envió parte de la muestra al Servicio de Anatomía Patológica, donde se clasificó histopatológicamente por dos patólogos. De acuerdo con los criterios histológicos que establece la WHO para clasificar los gliomas, se examina en las biopsias i) núcleos atípicos, ii) mitosis, iii) proliferación endotelial y iv) necrosis. En el caso de los Gbm deben existir al menos 3 de estas características, siendo las más representativas la presencia de marcada proliferación vascular y necrosis. Para los tumores de más bajo grado las biopsias pueden presentar ninguna, una o dos características, dependiendo si son grado I, II o III respectivamente. Para el análisis estadístico de los pacientes se utilizo la base de datos del Servicio de Neurocirugía del Instituto de Neurocirugía Dr. Asenjo (INCA).

Las biopsias tumorales se obtuvieron por procedimientos quirúrgicos de pacientes con Gbm en el INCA. Después de su extracción, una porción de la muestra se introdujo en medio RNAlater para la purificación de ARN total y otra parte se mantuvo en medio DMEM-F12 suplementado con suero bovino fetal (SBF) $15 \%$ y $1 \%$ antibióticos a $4^{\circ} \mathrm{C}$ para la preparación del cultivo primario de células. El tejido tumoral se sometió a digestión con $50 \mathrm{mg}$ de Colagenasa tipo IV (205 unidades/ $\mathrm{mg}$ ) durante $20 \mathrm{~min}$ con agitación constante a $37^{\circ} \mathrm{C}$. Para detener la digestión se agregó $10 \%$ SBF. Las células recolectadas fueron sedimentadas por 
centrifugación a $1.000 \mathrm{x}$ g durante 5 min y suspendidas en $5 \mathrm{ml}$ de medio DMEM-F12 suplementado con 20\% SBF y antibióticos $1 \%$ en condiciones de $37^{\circ} \mathrm{C}$ y $5 \%$ de $\mathrm{CO}_{2}$. Para determinar la expresión de los transportadores $\mathrm{ABC}$ se realizaron ensayos de RT-PCR, para ello se extrajo el ARN total desde el tejido tumoral o las células en cultivo primario utilizando el reactivo Trizol ${ }^{\circledR}$ (Invitrogen, USA). La primera hebra de ADNc fue sintetizada desde $1 \mu \mathrm{g}$ de ARN total usando $50 \mathrm{U}$ de $M-M u L V$ Reverse Transcriptase (Stratagene, USA) y oligo dT18 siguiendo las recomendaciones de los fabricantes. Las amplificaciones de PCR se realizaron usando $1 \mu \mathrm{lde}$ ADNc, tampón de PCR 1x, 1,5mM Mg ${ }^{+2}, 0,4 \mathrm{mM}$ dNTP's, 1,25U Taq ADN polimerasa (Invitrogen, USA) y $0,5 \mu \mathrm{M}$ de los oligonucleótidos específicos ${ }^{15}$. Para el control de la eficiencia de la transcripción reversa y PCR se realizó la amplificación del ADNc del gen $\beta$-actina. Los productos de amplificación se fraccionaron en geles de agarosa al 1,5\% y fueron visualizados por tinción con bromuro de etidio. Las imágenes fueron analizadas usando el programa GelPro31. Los partidores utilizados en este estudio fueron derivados desde las secuencias de los mensajeros de humano anotados en la base de datos GenBank ${ }^{\circledR}$ del Instituto Nacional de Salud de EEUU (NIH). Para los experimentos de western blot, los extractos celulares obtenidos de los cultivos fueron separados en geles de poliacrilamida al $10 \%$ y luego transferidos a membranas de nitrocelulosa, posteriormente sometidos a la detección inmunológica por $1 \mathrm{hr}$ a una dilución de 1:1.000 con anticuerpos específicos anti-Mrp1 (sc-18835C-19, Santa Cruz, USA), anti-Pgp (sc-55510, Santa Cruz, USA) y anti-ABCG2 (sc- 58222, Santa Cruz, USA) y como control se utilizó anti $\beta$-actina HPR (sc47778, Santa Cruz, USA). Después de los lavados las membranas fueron incubadas con el anticuerpo secundario anti-mouse IgG acoplado a peroxidasa (DAKO, Dinamarca) a una dilución de 1:5.000. El revelado se llevó a cabo utilizando kit de Bioluminiscencia ECL (Thermo scientific) y el equipo Ultra Lum. Para los ensayos de inmunohistoquímica, los tejidos de cerebro fueron fijados en Bouin, incluidos en parafina y seccionados en cortes de $5 \mu \mathrm{m}$. La inmunodetección se realizó secuencialmente desparafinando y rehidratando los cortes, luego incubados con $10 \mathrm{mM}$ citrato de sodio $(\mathrm{pH} 6,0)$ por $30 \mathrm{~min}$, peróxido de hidrogeno (70\% metanol, $3 \%$ perhidrol) por $5 \mathrm{~min}$, y bloqueados con PBS $1 \mathrm{X}$ conteniendo $1 \%$ BSA, $0,3 \%$ Tritón X-100 y $5 \%$ le- che descremada por 30 min a temperatura ambiente. Los cortes fueron incubados con el anticuerpo monoclonal anti MRP1, ABCG2 o MDR1 (Santa Cruz Biotechnology, USA) en solución de bloqueo toda la noche a $4^{\circ} \mathrm{C}$. Luego, los cortes fueron lavados tres veces con PBS1x por 5 min y revelados usando el sistema LSAB+System-HRP (DakoCytomation, USA ${ }^{16}$. Para medir la actividad de MRP1, $2 \times 10^{5}$ células de cultivo primario fueron sembradas en placas de 24 pocillos y pre-incubadas en medio libre de suero por $6 \mathrm{~h}$ a $37^{\circ} \mathrm{C}$. Posteriormente, fueron cargadas con CFDA ( $500 \mathrm{nM}$ ) por $15 \mathrm{~min}$, lavadas 3 veces con PBS $1 \mathrm{X}$ e incubadas por $30 \mathrm{~min}$, con medio libre de suero control o suplementado con MK571 $(20 \mu \mathrm{M})$. Las células fueron lavadas 3 veces con PBS $1 X$ frío y lisadas con PBS $1 X$ - 0,4\% Tritón $\mathrm{X}-100$. Las muestras se diluyeron 10 veces en PBS $1 \mathrm{X}$ a temperatura ambiente, la solución resultante se traspasó a una cubeta de cuarzo de paso óptico de $1 \mathrm{~cm}$. La fluorescencia en los extractos celulares fue medida en un espectrofluorímetro (Perkin Elmer LS-55) utilizando una longitud de onda de excitación y de emisión de $488 \mathrm{~nm}$ y $530 \mathrm{~nm}$ respectivamente. Este ensayo fue normalizado con respecto a la concentración total de proteínas. En el caso de los ensayos de viabilidad celular, las células fueron sembradas en placas de 96 pocillos a una concentración aproximada de $1 \times 10^{4}$ células por pocillo. Posteriormente, fueron incubadas por 24 hrs con medio DMEM-F12 sólo (sin tratamiento), con vincristina $100 \mathrm{nM}$, y/o vincristina $100 \mathrm{nM}$ con el inhibidor específico para el transportador, MK571 $(20 \mu \mathrm{M})$. Luego las células fueron incubadas con $5 \mathrm{mg} / \mathrm{ml}$ de MTT en medio de cultivo por 1 hora, posteriormente los cristales de formazan fueron lisados con $100 \mu \mathrm{l}$ de DMSO y se midió la absorbancia del compuesto producido a $550 \mathrm{~nm}$ en un lector de placas.

Los datos son presentados como promedio \pm desviación estándar. Los grupos fueron comparados mediante test $t$ de Student. Se consideraron diferencias significativas estadísticamente cuando el valor de $P$ fue menor de 0,05 . La sobrevida se analizó mediante los métodos Winstat 2.0 (software) y Excel 2007.

\section{Resultados}

De acuerdo a los resultados estadísticos en el INCA, el número de Gbm registrados ha ido aumentando, en el año 2007 fueron 14 casos diag- 
nosticados, 28 casos en 2008 y 31 en 2009. De estos 73 registros sólo 7 pacientes se mantienen vivos a la fecha, representando 9,6\%, con un rango de seguimiento de 0 a 43 meses (Tabla 1). Debemos considerar que el número de fallecidos se ha mantenido contante entre estos años, representando una alta tasa de mortalidad de 92,9\%, 89,3\% y $87,1 \%$ (Tabla 1). Siendo la mediana de sobrevida de la serie total de 6 meses y las medianas de sobrevivencia al año de 3,9, a los dos años de 5,7 y a los tres años de 5,9 (Tabla 1). Estos pacientes presentan un promedio de edad de 57,$8 ; 53,5$ y 52,8 años entre 2007 y 2009 , respectivamente, con un rango de edad de 5-73 años (Tabla 1). El gráfico de la Figura 1 indica que al año la sobrevivencia de los pacientes del INCA alcanza $27 \%$ y a los dos y tres años sólo $10 \%$ y $6 \%$, respectivamente. También podemos visualizar en la Tabla 2 que

Tabla 1. Datos estadísticos de pacientes diagnosticados con glioblastoma multiforme entre los años 2007 y 2009 en el Instituto de Neurocirugía Asenjo (INCA)

\begin{tabular}{|lccc|}
\hline Datos pacientes INCA & $\mathbf{2 0 0 7}$ & $\mathbf{2 0 0 8}$ & $\mathbf{2 0 0 9}$ \\
\hline N $^{\circ}$ casos de novo (2007-2009) & 14 & 28 & 31 \\
\hline N $^{\circ}$ fallecidos & 13 & 25 & 27 \\
\hline Edad media al diagnóstico & 57,8 & 53,5 & 52,8 \\
\hline Rango de edad & $39-64$ & $5-73$ & $7-73$ \\
Tasa hombre/mujer & 5,5 & 0,9 & 1,3 \\
Rango de supervivencia (meses) & $0-43$ & $0-31$ & $0-22$ \\
Quimioterapia & 0 & 0 & 0 \\
Radioterapia & 0 & 0 & 0 \\
\hline Re-operados & 2 & 0 & 0 \\
\hline
\end{tabular}

Los datos se analizaron estadísticamente usando los programas Winstat 2.0 (software) y Excel 2007.

Mediana de supervivencia de la serie: 6,0 meses.

Mediana de supervivencia al año: 3,9 meses.

Mediana de supervivencia a los dos años: 5,7 meses.

Mediana de supervivencia a los tres años: 5,9 meses.

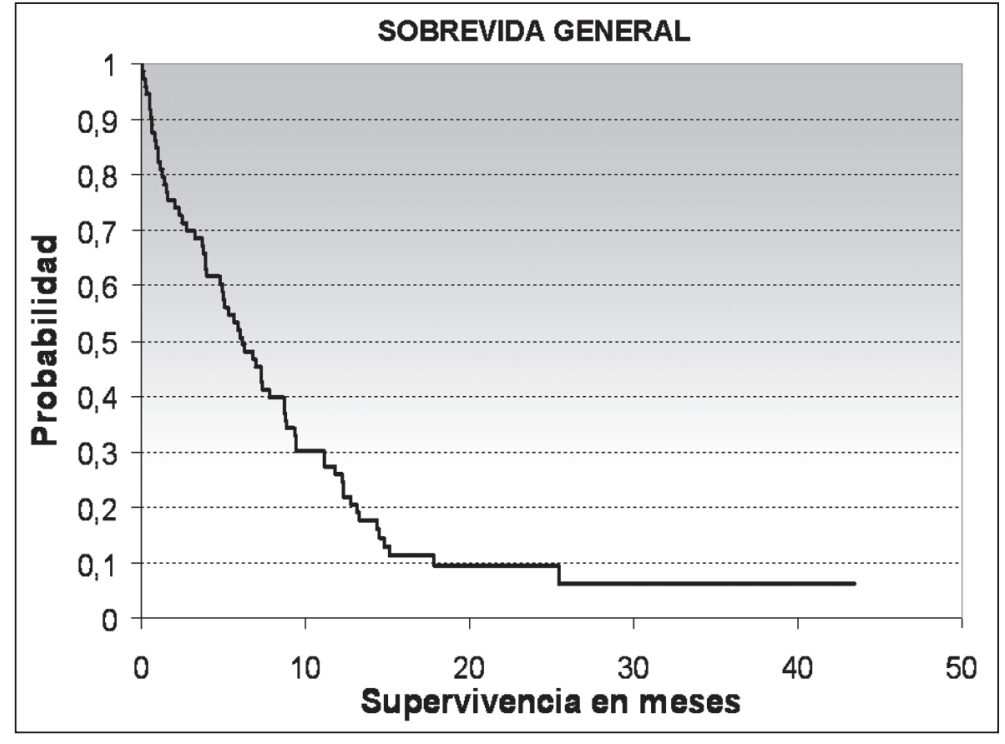

Figura 1. Sobrevida de pacientes registrados y fallecidos con glioblastoma multiforme en el Instituto de Neurocirugía Dr. Asenjo (INCA) entre los años 2007 y 2009. El gráfico de supervivencia de los pacientes entre los años 2007 y 2009 se realizó usando la base de datos del INCA y el programa Winstat 2.0 (software). 
Resistencia a quimioterapia en glioblastoma multiforme - C. Quezada et al

Tabla 2. Total de registros de tumores en el Instituto de Neurocirugía Asenjo (INCA), Santiago, Chile, entre los años 2007 y 2009

\begin{tabular}{|c|c|c|c|c|}
\hline Tipo de tumor & $\%$ del total 2007 & $\%$ del total 2008 & \% del total 2009 & $\%$ totales \\
\hline Oligodendroglioma & 2,47 & 1,48 & 4,58 & 2,82 \\
\hline Hemangiopericitoma & 0 & 1,73 & 0,51 & 0,75 \\
\hline Tumor de hipófisis & 21,23 & 26,91 & 23,41 & 23,78 \\
\hline Meningeoma & 18,3 & 19,51 & 18,07 & 18,56 \\
\hline Astrocitoma grado 1 & 5,93 & 2,72 & 4,07 & 4,23 \\
\hline Astrocitoma grado 2 & 4,20 & 3,70 & 4,07 & 3,98 \\
\hline Astrocitoma grado 3 & 3,95 & 1,48 & 3,05 & 2,82 \\
\hline Glioblastoma & 3,46 & 6,91 & 7,89 & 6,05 \\
\hline Plasmocitoma & 0,25 & 0,99 & 0,25 & 0,50 \\
\hline Ependimoma & 3,20 & 5,19 & 2,80 & 3,73 \\
\hline Metástasis & 4,20 & 3,70 & 4,07 & 3,98 \\
\hline PNET & 0 & 0,74 & 0,76 & 0,50 \\
\hline Maligno & 0 & 1,73 & 0 & 0,58 \\
\hline Schwanoma & 7,90 & 5,19 & 3,56 & 5,55 \\
\hline Linfoma & 2,47 & 0,99 & 0,51 & 1,33 \\
\hline Ganglioglioma & 3,95 & 2,96 & 2,29 & 3,07 \\
\hline Sarcoma & 0,99 & 0,99 & 0,76 & 0,91 \\
\hline Lipoma & 2,47 & 1,73 & 3,56 & 2,57 \\
\hline Quiste epidermoide & 2,47 & 2,47 & 2,04 & 2,32 \\
\hline Meduloblastoma & 2,22 & 2,47 & 1,78 & 2,15 \\
\hline Hemangioblastoma & 1,23 & 1,98 & 0,51 & 1,24 \\
\hline Teratoma & 0 & 0,25 & 0,25 & 0,17 \\
\hline Papiloma & 1,48 & 0,74 & 1,27 & 1,16 \\
\hline Craneofaringeoma & 3,46 & 1,23 & 4,07 & 2,90 \\
\hline Germinoma & 0,74 & 0,49 & 1,53 & 0,91 \\
\hline Osteoma & 0 & 0,25 & 0 & 0,08 \\
\hline Miofibroblástico & 0 & 0,25 & 0 & 0,08 \\
\hline Cordoma & 0,25 & 0,25 & 1,02 & 0,50 \\
\hline Neurofibroma plexiforme & 0,99 & 0,49 & 0,76 & 0,75 \\
\hline Carcinoma & 0,25 & 0,49 & 0,25 & 0,33 \\
\hline Teratoide rabdoide & 0,25 & 0 & 0,25 & 0,17 \\
\hline Epitelioma & 0,25 & 0 & 0 & 0,08 \\
\hline Paraganglioma & 0,74 & 0 & 0 & 0,25 \\
\hline Leiomioma & 0,74 & 0 & 0 & 0,25 \\
\hline Pineocitoma & 0 & 0 & 1,53 & 0,50 \\
\hline Retinoblastoma & 0 & 0 & 0,25 & 0,08 \\
\hline Osteoblastoma & 0 & 0 & 0,25 & 0,08 \\
\hline Total de pacientes & 395 & 405 & 393 & 1.193 \\
\hline
\end{tabular}

Los datos de los tumores cerebrales operados en el INCA fueron analizados estadísticamente utilizando el programa EXCEL 2007. 
los Gbm corresponden, en promedio, a 6,05\% del total de tumores registrados en este Instituto. Este porcentaje sobresale si lo comparamos con respecto a otros tipos de tumores operados, siendo los tumores de hipófisis y meningeomas los únicos de mayor proporción a los Gbm (Tabla 2).

Para realizar este estudio se estandarizó el cultivo primario de células de Gbm de humano en 18 biopsias. Las células una vez que se adhirieron a la placa comenzaron su crecimiento en forma radial aproximadamente luego de siete días (Figura 2A). Los cultivos primarios de las células de Gbm se caracterizaron por presentar una apariencia pleomórfica, con tendencia a la fusión entre sus prolongaciones que comenzó después de catorce días de realizado el cultivo (Figura 2B). Nosotros
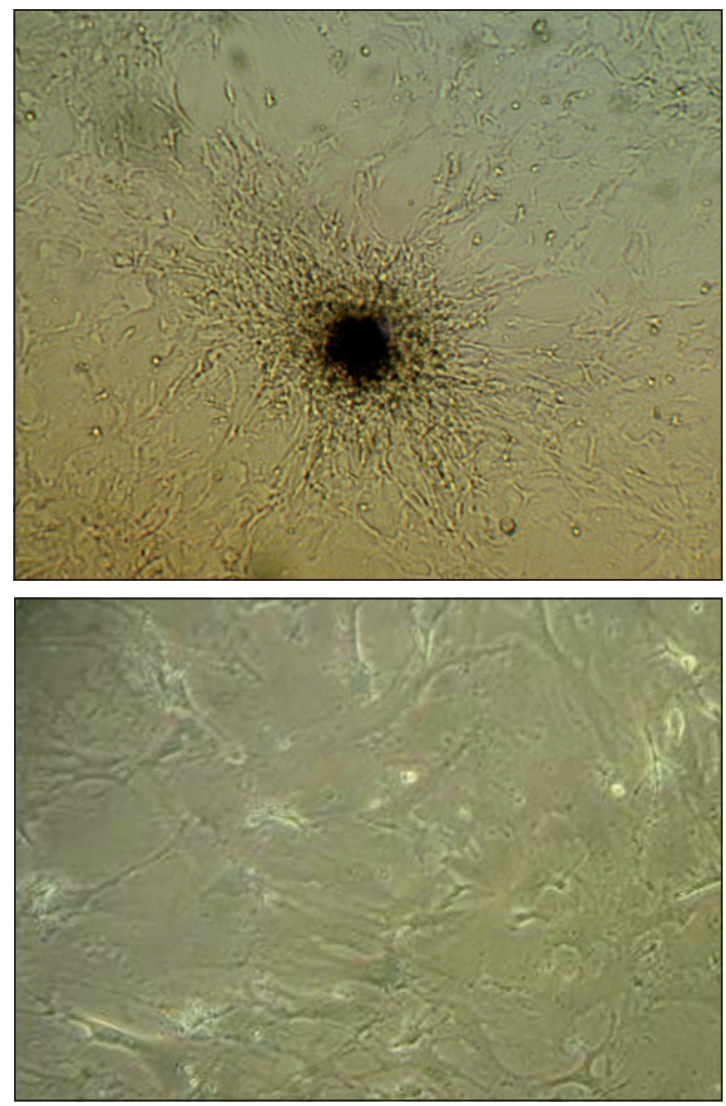

Figura 2. Cultivo primario de glioblastoma multiforme humano. Las células de Gbm fueron cultivados en medio DMEMF12 suplementado con suero bovino fetal a $37^{\circ} \mathrm{C}$ y $5 \% \mathrm{CO}_{2}$. A. Crecimiento radial de cultivo primario de $\mathrm{Gbm}$ humano a los primeros 7 días. B. Crecimiento pleomórfico luego de 14 días. Magnificación original 200X $(n=18)$. detectamos por RT-PCR y western blot la expresión de los transportadores Mrp1, P-gp (Mdr1) y ABCg2 (Figura 3A y 3B). En tejido y células de glioblastoma pudimos determinar una mayoritaria expresión del transportador Mrp1 con respecto a P-gp y ABCg2. La señal detectada para Mrp1 en los

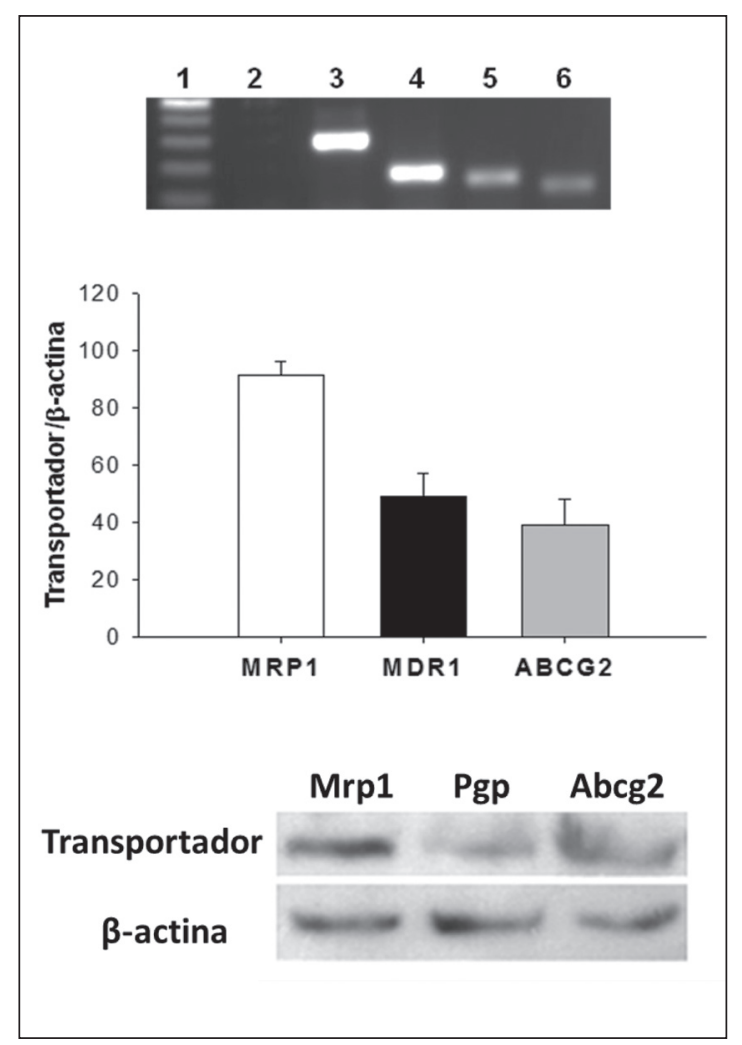

Figura 3. Expresión de los genes de transportadores $A B C$ en cultivo primario de glioblastoma humano. La expresión de los transportadores fue detectada mediante amplificación por RTPCR de los transcritos (A) y de las proteínas por western blot en células de cultivo primario de Gbm humano (B). (A) Arriba: Los productos de amplificación fueron fraccionados en un gel de agarosa al 1,5\%. Carril 1: St., estándar de tamaño molecular de $100 \mathrm{pb}$, carril 2: control negativo, carril 3: $\beta$-actina (300pb), carril 4: Mrp1 (200pb), carril 5: P-gp (159pb), carril 6: ABCg2 (150pb). Abajo: El gráfico representa el promedio de la razón entre la intensidad de fluorescencia del producto de amplificación de cada transportador vs $\beta$-actina. Los valores son el promedio \pm D.E. $(n=5) .{ }^{*}, P<0,01$ versus control. (B) Las proteínas de cultivo primario fueron separadas mediante electroforesis en geles de poliacrilamida al $10 \%$ y transferidas a membranas de nitrocelulosa, luego fueron incubadas con los anticuerpos anti-Mrp1, anti-P-gp, anti-ABCg2 y se reveló usando el sistema de quimioluminiscencia $\mathrm{ECL}$, como control se utilizó $\beta$-actina $(n=3)$. 
experimentos de inmunohistoquímica colocaliza con el marcador de células gliales (anticuerpo anti GFAP) y con células en proceso de proliferación celular (anticuerpo anti Ki-67) (Figura 4). Coherente con lo observado en los ensayos de expresión, la señal de Mrp1 fue claramente más intensa en la zona del tumor y levemente detectable en la región peritumoral. Al contrario, la marca de $\mathrm{P}$-gp y $\mathrm{ABCg} 2$ fueron homogéneas en toda la muestra, incluyendo tejido peritumoral y tumoral, y en esta última región la señal fue mucho menos intensa que la de Mrp1 (Figura 4). La funcionalidad de la proteína Mrp1 fue ensayada en células de Gbm cultivadas. Las células fueron cargadas con CFDA, el cual es un sustrato del transportador $\mathrm{Mrp}^{17} \mathrm{e}$ incubadas por un período de tiempo (30 $\mathrm{min})$ durante el cual este sustrato será expulsado de la célula. La actividad Mrp1 es evidente al utilizar un inhibidor de su actividad, MK571 ${ }^{18}$, lo cual provoca una acumulación del sustrato fluorescente intracelularmente (Figura 5).

Finalmente, nosotros evaluamos in vitro la
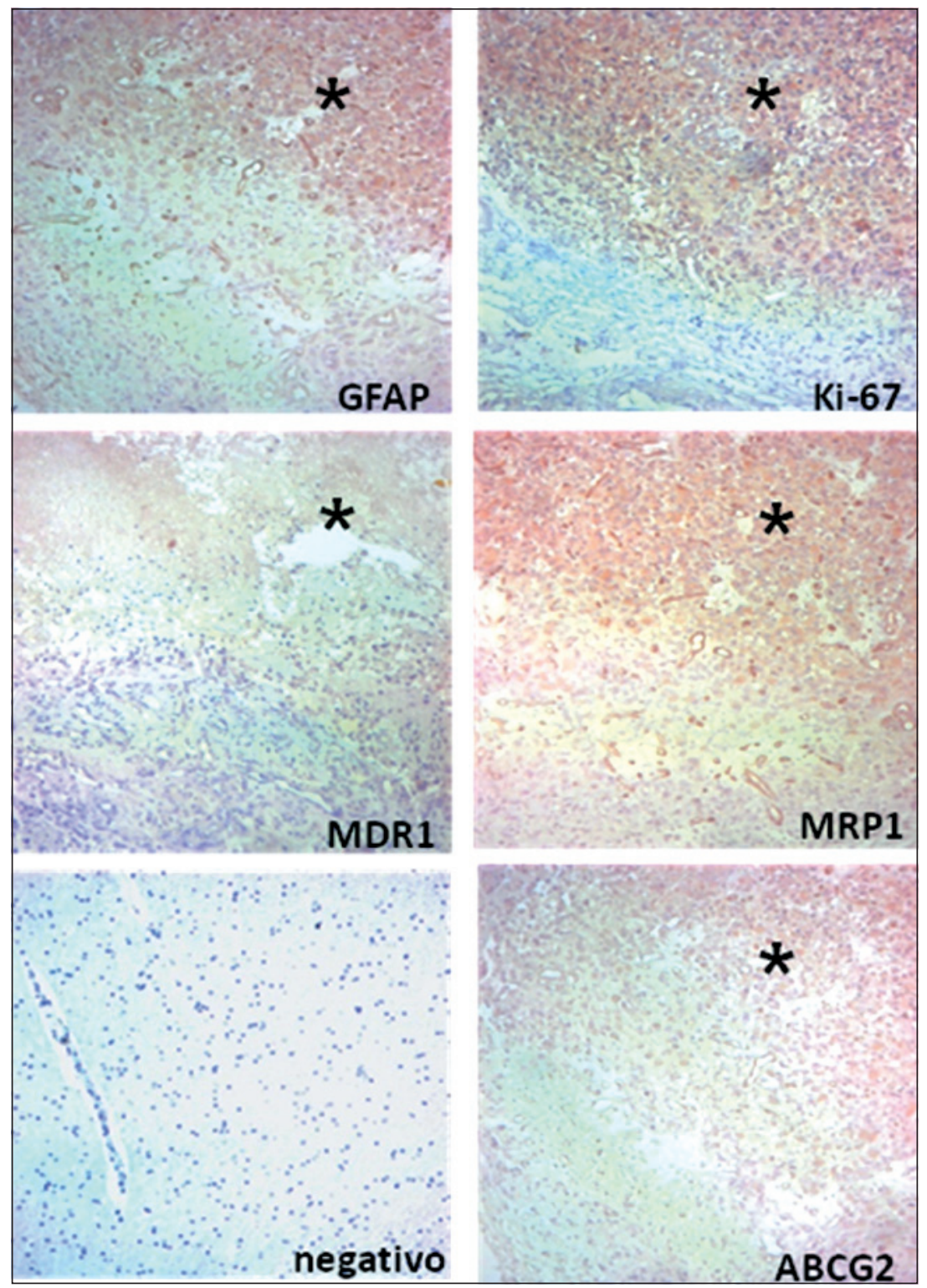

Figura 4. Expresión de transportadores $A B C$ en biopsias de cerebro humano. Los tejidos fueron obtenidos de cirugías de resección de glioblastoma multiformes y procesados para inmunohistoquímica. Los anticuerpos primarios anti -GFAP, -Ki-67, -Mrp1, -P-gp y -ABCg2 fueron utilizados en los diferentes cortes como se indica en cada imagen. La inmuno-detección fue revelada utilizando el substrato DAB para la actividad peroxidasa. Las muestras fueron también teñidas con hematoxilina. El control negativo no incluyó anticuerpo primario. Magnificación 200X. *indica la zona del tumor $(n=5)$. 
Tabla 3. Efecto de la inhibición de Mrp1 sobre la citotoxicidad de la droga vincristina en células de glioblastoma humano

\begin{tabular}{|c|c|c|c|}
\hline & s/tratamiento & Vincristina & Vincristina + MK571 \\
\hline Viabilidad celular & $100 \pm 7,65$ & $93 \pm 8,20$ & $58 \pm 6,5$ \\
\hline
\end{tabular}

Las células de glioblastoma humano fueron incubadas con medio DMEM-F12 solo (s/tratamiento), con vincristina 100 nM, y/o vincristina 100nM con el inhibidor MK571 $(20 \mu \mathrm{M})$. La viabilidad celular fue cuantificada después de 24 hrs de tratamiento usando el ensayo de reducción del colorante MTT $(n=5)$.

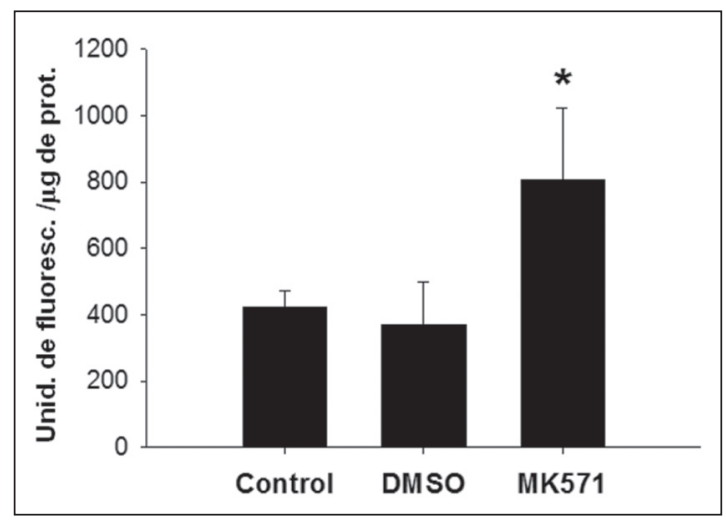

Figura 5. Actividad del transportador Mrp1 en células de cultivo primario de glioblastoma humano. Las células fueron cargadas con CFDA e incubadas por 30 min en ausencia o expuestas a DMSO $(0,1 \%)$ o MK571 (20 mM). La cantidad de CFDA que permanece intracelularmente se determinó midiendo la cantidad de fluorescencia en el lisado de células. ${ }^{*} \mathrm{P}<0,01(n=3)$.

quimiosensibilización de las células cancerosas utilizando un inhibidor del transportador Mrp1. Para ello las células del cultivo primario de Gbm fueron expuestas a vincristina, un sustrato de Mrp1, y se determinó el efecto sobre la viabilidad celular. La viabilidad celular disminuyó en 7,0\% aproximadamente, lo cual fue significativamente potenciado al exponer las células a vincristina y el inhibidor MK571 (Tabla 3).

\section{Discusión}

La incidencia de los tumores primarios de cerebro ha tenido un notorio incremento en las últimas décadas, cada año aproximadamente 14.000 personas padecen de cáncer cerebral a nivel mundial ${ }^{3,4}$. Nuestros análisis demuestran un aumento del porcentaje de casos de Gbm en la población estudiada sin encontrar variaciones del número total de pacientes diagnosticados con tumores cerebrales entre 2007 y 2009. Esto sugiere un aumento de la incidencia del Gbm ya que se ha descartado otros factores como mayor derivación al centro de neurocirugía involucrado en el estudio o diferencias en el diagnóstico. Además, el porcentaje de fallecidos por esta enfermedad en los años analizados sigue siendo dramáticamente alta.

A pesar de los avances en tecnologías de imágenes para la detección temprana del glioblastoma, las mejoras en el tratamiento han sido de limitada efectividad. En Chile, actualmente el manejo de los pacientes con tumores cerebrales es la cirugía y luego radioterapia con seguimiento de la evolución a través de tomografía axial computarizada (TAC) y/o resonancia nuclear magnética (RNM) de cerebro. Sin embargo, debemos considerar que la extracción de los tumores cerebrales, generalmente es incompleta ya que está limitada por el daño neurológico y la localización del tumor, lo cual conlleva a que los pacientes deriven en reincidencia del tumor o transformación maligna ${ }^{19}$. La OMS aprobó el uso de temozolomida en la quimioterapia del Gbm luego de los estudios clínicos publicados el año 2005 que demostraron un aumento de la media de sobrevida a 14,6 meses en los pacientes sometidos a radioterapia y quimioterapia en comparación a aquellos que sólo recibieron radioterapia post cirugía cuya sobrevida fue de 12,1 meses ${ }^{20}$. Hasta ahora, la quimioterapia no ha sido un aporte significativo al tratamiento de los pacientes afectados por tumores cerebrales malignos de alto grado, a pesar de tener la ventaja de ser un método no invasivo y que pudiera tener mucho menos complicaciones comparado a la cirugía y radioterapia. Se han utilizado nuevas generaciones de fármacos en clínica los cuales han demostrado una baja efectividad (15-30\% de aumento de la sobrevida) para tratar tumores de 
alto grado. Entre estos fármacos el que se utiliza en muchos de nuestros centros médicos en Chile es la temozolomida (Temodal $\left.{ }^{\circledR}\right)$, el cual es un agente alquilante del ADN. Sin embargo, se ha demostrado que las células de Gbm multiforme poseen resistencia intrínseca a la metilación del $\mathrm{ADN}$ debido a la elevada actividad de la enzima $O^{6}$ methyguanine-DNA-methyltransferase (MGMT) en estas células ${ }^{21,22}$. Además, se ha establecido que el pronóstico de sobrevida de los pacientes tratados con temozolomida también está limitado al grado de metilación del promotor del gen $\mathrm{MGMT}^{23-25}$. Esta resistencia de las células de Gbm a agentes que metilan el ADN se conoce como "resistencia particular a drogas". Además, estas células son extremadamente quimoresistentes a un amplio espectro de fármacos antitumorales lo cual se conoce como "resistencia múltiple a drogas" y está dado por la actividad de uno o más transportadores de la familia ABC. Es sabido que la actividad de los transportadores de resistencia múltiple a drogas en las células de la microvasculatura cerebral permite la actividad de barrera al paso de moléculas al cerebro, aunque cabe destacar que la mayor resistencia en el cáncer cerebral de alto grado está dada por los mecanismos de resistencia en el glioma mismo, ya que la BHE aumenta su permeabilidad debido al rompimiento de las uniones estrechas entre sus células endoteliales ${ }^{26,27}$. Por lo tanto, una estrategia para quimiosensibilizar este tipo de células cancerosas es reconocer los transportadores que confieren la resistencia múltiple a fármacos antitumorales, y utilizar inhibidores de su actividad como coadyuvantes al tratamiento.

Nuestros resultados demuestran, al estudiar el fenómeno de resistencia múltiple a drogas en tejidos y cultivo primario de los Gbm, la alta expresión y colocalización de Mrp1 en zonas de crecimiento tumoral. Asimismo, la actividad de este transportador fue confirmada en estas células cancerosas y es susceptible de ser inhibido utilizando un bloqueador específico. Otros estudios utilizando análisis citofluorimétrico de diferentes especímenes de gliomas y sus cultivos primarios, también indican que existe un alto nivel de expresión de la proteína Mrp1, moderados niveles de Mrp5 y bajos niveles de Mrp3, Mrp2 y P-gp ${ }^{12,13}$. Además, estudios en líneas celulares de gliomas corroboran un predominante contenido de miembros de la subfamilia MRP, particularmente Mrp $1^{14}$. Interesantemente, demostramos in vitro que al exponer las células de cultivo primario de Gbm a vincristina en conjunto con MK571, inhibidor de Mrp1, la viabilidad celular disminuyó notoriamente. Actualmente, existen inhibidores de alta selectividad para Mrp1, pero hasta ahora sólo sulindac ha sido demostrado ser seguro en estudios en fase clínica. Este fármaco está siendo evaluado en ensayos clínicos para la terapia conjunta con fármacos antitumorales en cáncer de mama y melanoma ${ }^{28}$, debido a que al igual que los gliomas de alto grado presentan la particularidad de tener una actividad de Mrp1 aumentada. También existen moléculas inhibidoras del transportador P-gp y que también pueden inhibir a Mrp1. Tal es el caso de CBT-1 and biricodar los cuales también han sido evaluados en estudios en fase clínica ${ }^{29,30}$.

Actualmente, la mortalidad de los pacientes con Gbm continúa siendo extremadamente alta y por lo tanto, existe una demanda por nuevas alternativas de tratamiento. Con este objetivo está en evaluación el uso de inhibidores de la angiogénesis (bevacizumab) en pacientes con $\mathrm{Gbm}^{31}$. Nosotros postulamos que las células de Gbm podrían ser quimiosensibilizadas utilizando inhibidores de Mrp1, lo cual sería una nueva alternativa terapéutica para ser evaluada en fase clínica y que podría mejorar la eficiencia de la quimioterapia contra este tipo de tumor.

\section{Referencias}

1. Jemal A, Siegel R, Ward E, Hao Y, Xu J, Murray T, et al. Cancer statistics. Cancer J Clin 2008; 58: 71-96.

2. Díaz V, Yáñez A, Ponce C, Villegas, Pasten J. Tendencia de la mortalidad por tumores cerebrales malignos en Chile. Análisis de tasas. Rev Chil Neuro-Psiquiat 2006; 44: 263-70.

3. Sawyer A, Piepmeier J, Saltzman W. New methods for direct delivery of chemotherapy for treating brain tumors. Yale J Biol Med 2006; 79: 141-52.

4. Brandes MM. State-of-the-art treatment of high-grade brain tumors. Semin Oncol 2003; 30 (6 Suppl 19): 4-9.

5. Valera E, Lucio-Eterovic A, Neder L, Scrideli C, Machado $\mathrm{H}$, Carlotti-Junior C, et al. Quantitative PCR analysis of the expression profile of genes related to multiple drug resistance in tumors of the central nervous system. J Neurooncol 2007; 85: 1-10.

6. Mladkova N, Chakravarti A. Molecular Profiling in Glioblastoma: Prelude to Personalized Treatment. Current Oncology Reports 2009; 11: 53-61. 
7. Sharom FJ. ABC multidrug transporters: structure, function and role in chemoresistance. Pharmacogenomics 2008; 9: 105-27.

8. Loscher W, Potschka H. Drug resistance in brain diseases and the role of drug efflux transporters. Nat Rev Neurosci 2005; 6: 591-602.

9. Muldoon LL, Soussain C, Jahnke K, Johanson C, Siegal T, Smith QR, et al. Chemotherapy delivery issues in central nervous system malignancy: a reality check. J Clin Oncol 2007; 25: 2295-305.

10. Bredel M, Zentner J. Brain-tumour drug resistance: the bare essentials. Lancet Oncology 2002; 3: 397-406.

11. Szakács G, Paterson JK, Ludwig JA, Booth-Genthe C, Gottesman MM. Targeting multidrug resistance in cancer. Nat Rev Drug Discov 2006; 5: 219-34.

12. Calatozzolo C, Gelati M, Ciusani E, Sciacca FL, Pollo B, Cajola L, et al. Expression of drug resistance proteins Pgp, MRP1, MRP3, MRP5and GST-pi in human glioma. J Neurooncol 2005; 74: 113-21.

13. Decleves X, Amiel A, Delattre JY, Schermann JM. Role of $\mathrm{ABC}$ transporters in the chemoresistance of human gliomas. Curr Cancer Drug Targets 2006; 6: 433-45.

14. Matsumoto Y, Miyakei K, Kunishio K, Tamiya T, Seigo $\mathrm{N}$. Reduction of expression of the multidrug resistance protein (MRP) 1 in glioma cells by antisense phosphorothioate oligonucleotides. J Med Invest 2004; 51: 194-201.

15. Quezada C, Garrido W, González- Oyarzún M, Rauch MC, Salas M, San Martín R, et al. Effect of tacrolimus on activity and expression of Pgp and ABCA5 proteins in hematoencephalic barrier cells. Biol Pharm Bull 2008; 31: 1911-6.

16. Valladares D, Quezada C, Montecinos P, Concha II, Yáñez AJ, Sobrevia L, et al. Adenosine A(2B) receptor mediates an increase on VEGF-A production in rat kidney glomeruli. Biochem Biophys Res Commun 2008; 366: 180-5.

17. Van Der Deen M, Homan S, Timmer-Bosscha H, Scheper R, Timens W, Postma D, et al. Effect of COPD treatments on MRP1-mediated transport in bronchial epithelial cells. Int J Chron Obstruct Pulmon Dis 2008; 3: 469-75.

18. Gekeler V, Ise W, Sanders KH, Ulrich WR, Beck J. The leukotriene LTD4 receptor antagonist MK571 specifically modulates MRP associated multidrug resistance. Biochem Biophys Res Commun 1995; 208: 45-52.

19. Lu C, Shervington A. Chemoresistance in gliomas. Mol Cell Biochem 2008; 312: 71-80

20. Stupp R, van den Bent MJ, Hegi ME. Optimal role of temozolomide in the treatment of malignant gliomas. Curr Neurol Neurosci Rep 2005; 5: 198-206.
21. Hegi ME, Liu L, Herman JG. Correlation of $\mathrm{O}^{6}$ methylguanine methyltransferase (MGMT) promoter methylation with clinical outcomes in glioblastoma and clinical strategies to modulate MGMT activity. J Clin Oncol 2008; 26: 4189-99.

22. Nagane M, Kobayashi K, Ohnishi A, Shimizu S, Shiokawa Y. Prognostic significance of $\mathrm{O}^{6}$-methylguanine-DNA methyltransferase protein expression in patients with recurrent glioblastoma treated with temozolomide. Jpn J Clin Oncol 2007; 37: 897-906.

23. Brandes AA, Tosoni A, Cavallo G, Bertorelle R, Gioia V, Franceschi E, et al. Temozolomide 3 weeks on and 1 week off as first-line therapy for recurrent glioblastoma: phase II study from gruppo italiano cooperativo di neurooncología (GICNO). Br J Cancer 2006; 95: 1155-60.

24. Motomura K, Natsume A, Kishida Y, Higashi H, Kondo Y, Nakasu Y, et al. Benefits of interferon- $\beta$ and temozolomide combination therapy for newly diagnosed primary glioblastoma with the unmethylated MGMT promoter: a multicenter study. Cancer 2010.

25. van den Bent MJ, Dubbink HJ, Sanson M, van der LeeHaarloo CR, Hegi M, Jeuken JW, et al. MGMT promoter methylation is prognostic but not predictive for outcome to adjuvant PCV chemotherapy in anaplastic oligodendroglial tumors: a report from EORTC Brain Tumor Group Study 26951. J Clin Oncol 2009; 27: 5881-6.

26. Lee SW, Kim WJ, Park JA, Choi YK, Kwon YW, Kim KW. Blood-brain barrier interfaces and brain tumors. Arch Pharm Res 2006; 29: 265-75.

27. Hawkins BT, Davis TP. The blood-brain barrier/neurovascular unit in health and disease. Pharmacol Rev 2005; 57: 173-185.

28. O'Connor R, O'Leary M, Ballot J, Collins CD, Kinsella P, Mager DE, et al. Crown J. A phase I clinical and pharmacokinetic study of the multi-drug resistance protein-1 (MRP-1) inhibitor sulindac, in combination with epirubicin in patients with advanced cancer. Cancer Chemother. Pharmacol 2007; 59: 79-87.

29. Peck RA, Hewett J, Harding MW. Phase I and pharmacokinetic study of the novel MDR1 and MRP1 inhibitor biricodar administered alone and in combination with doxorubicin. J Clin Oncol 2001; 19: 3130-41.

30. Robey RW, Shukla S, Finley EM. Inhibition of P-glycoprotein $(\mathrm{ABCB} 1)$ - and multidrug resistance-associated protein 1 (ABCC1)-mediated transport by the orally administered inhibitor, CBT- 1(R). Biochem Pharmacol 2008; 75:1302-1312.

31. Peak SJ, Levin VA. Role of bevacizumab therapy in the management of glioblastoma. Cancer Manag Res 2010; 2: 97-104. 


\section{Referencias}

1. Martin W, Brynes S. Chilean Miners and Biomedical Research. A Modest Proposal. Am J Respir Crit Care Med 2010; 182: 1459-64.

2. Sobradillo P, Pozo F, Agustí A. Medicina P4: el futuro a la vuelta de la esquina. Arch Bronconeumol 2011; 47: 35-40.

Correspondencia a: Dr. Rafael Silva O.

Dos Norte 360, Talca. Fono: 71-412716 Fax: 71-209306

E-mail: rafaelsilvao@gmail.com 